\title{
A HYBRID BACKWARD-FORWARD METHOD FOR INTERACTIVE REFLECTIONS
}

\author{
Chunhui Mei, Voicu Popescu, and Elisha Sacks \\ Computer Science Department, Purdue University, 305 N University Street, West-Lafayette, USA \\ meich@cs.purdue.edu,popescu@cs.purdue.edu,eps@cs.purdue.edu
}

Keywords: Reflection rendering, interactive frame rates, projection, ray-tracing, $\mathrm{k}-\mathrm{d}$ tree, continuous 3-ray camera.

Abstract: $\quad$ We propose a two phase hybrid reflection rendering method based on approximating the reflected rays with a set of simple cameras modeled as continuous 3-ray cameras. In the first, "backward", phase, the view volume of each simple camera is intersected with a hierarchical subdivision of the scene to find the geometry it encompasses. In the second, "forward", phase the geometry is projected with the simple camera. Since the shape and topology of reflected triangles is complex, point based rendering is adopted to reconstruct the reflection. The hybrid method is efficient since it combines advantages of backward and forward techniques: there are two orders of magnitude fewer simple cameras than reflected rays, the hierarchical scene subdivision implements fast view volume culling for each of the simple cameras, and the reflection piece corresponding to each simple camera is computed efficiently in feed-forward fashion.

\section{INTRODUCTION}

Reflective surfaces are the first to draw the attention of a user that visually explores a 3D scene. In addition to intriguing aesthetic quality, reflections bring concrete contributions to the user's understanding of the scene, revealing surface properties and the relative position of objects. Unfortunately, rendering reflections is challenging. The difficulty comes from the fact that every reflective surface is essentially a portal into a world that is potentially more complex than the directly observed scene. In the reflected scene the rules of image formation are substantially more complex than those in the case of single perspective: a 3D point can have more than one projection, straight lines project to curves, some surfaces are magnified and others minified to the extreme. Much work has been devoted to rendering reflections, but no technique exists that renders accurate reflections on general surfaces at interactive rates. We group prior techniques in four categories.

Ray tracing techniques search for scene geometry along desired view and reflected rays. Such techniques produce breathtaking reflections, but the backward mapping from the output pixels to input geometry is inefficient, and ray tracing is not the approach of choice in interactive graphics. The feed-forward graphics pipeline, with the main stages of projection and rasterization, has proven to be the best suited for computer graphics applications where efficiency is at a premium. However, rendering reflections with the feed-forward approach requires solving the difficult problem of projecting reflected vertices. For a general reflector surface there is no closed form solution to the problem of finding the image plane location of a reflected 3D point. Feedforward techniques were developed for planar and convex reflectors, cases when the problem of projecting reflected vertices is tractable.

Reflected-scene approximation techniques intersect reflected rays with a simpler version of the scene, in the interest of efficiency. A prime example is environment mapped reflections, where the reflected scene is replaced with a distant color panorama. Less drastic approaches simplify the scene using billboards or depth map impostors. The disadvantages of the approach are loss of accuracy and additional modeling burden.

Image-based rendering (IBR) techniques employ pre-acquired or pre-rendered reference reflections to reconstruct the reflections in the desired view. The forte of these techniques is the visual realism inherited from photographs or from reference images elaborately rendered offline. Disadvantages include limited support for highly reflective surfaces, which require a high sampling rate, and limited support for dynamic scenes, where reference reflections become obsolete. 

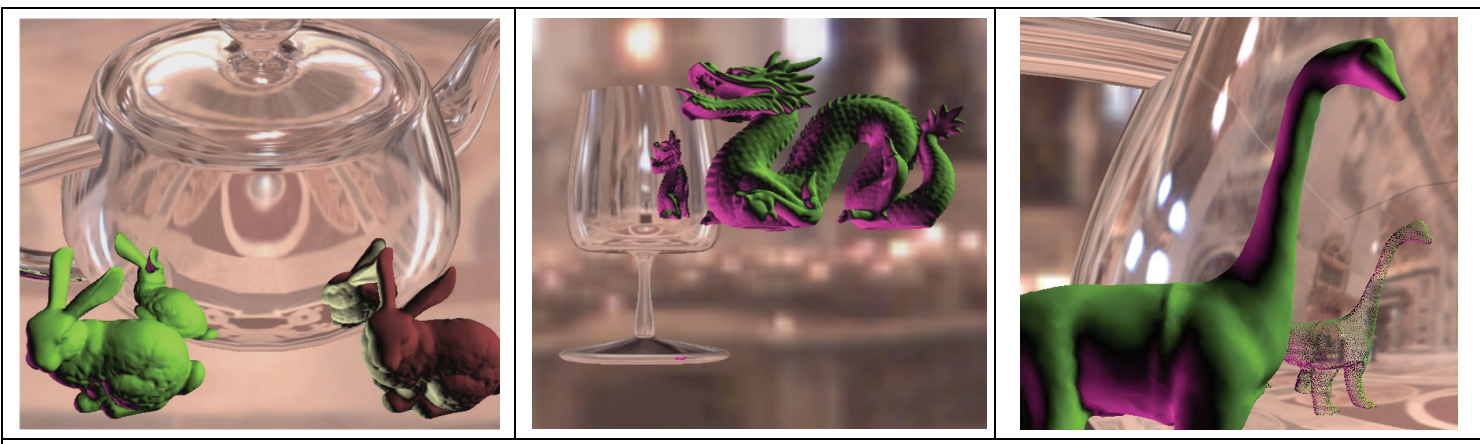

Figure 1: Objects reflected with our technique. The reflector complexity [thousands of triangles], reflected objects total complexity [thousands of vertices], and average frame rate [fps] are 4, 74, and 5 for the left image, and 2,100, and 4 for the middle image. The reflection of the objects is rendered in point-based fashion, as illustrated in the right image.
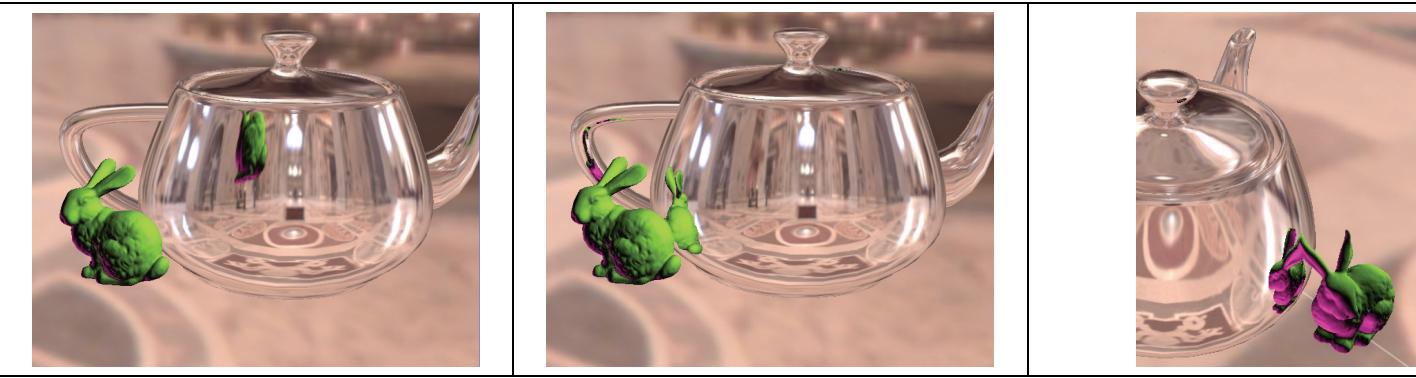

Figure 2: Comparison to environment mapping. Environment mapping (left) is inaccurate for objects close to the reflector. Our technique (middle) produces correct reflections even if the object intersects the reflector (right).

In this paper we describe a hybrid technique that combines elements characteristic to ray tracing with feed-forward rendering. The desired view and the reflectors in the scene define a set of reflected rays for each frame. Depending on frame resolution and on how much of the frame is covered by reflectors, the set of reflected rays can contain hundreds of thousands of rays. Our technique approximates these rays with a few thousand simple cameras by taking advantage of the local coherence exhibited by the ray set. A simple camera is an atomic camera that has an efficient projection function. A simple camera could be modeled as a planar pinhole camera, but the reflected rays are not concurrent and forcing them through a pinhole introduces large errors. We use instead a continuous 3-ray camera (C3RC) [PDM*06], a non-pinhole model that interpolates between 3 given rays. The C3RC is a generalization of the general linear camera [YM04a], with the advantage of projection continuity across shared edges of adjacent cameras.

In a first phase, the view volume of each C3RC is intersected with the scene to find the geometry it encompasses. This backward mapping phase is common to ray tracing techniques, and we accelerate it using a kd-tree [Ben79] scene subdivision. The kdtree ensures efficient frustum culling for the C3RCs.

In a second phase, the reflection is computed in feed-forward fashion. Each simple camera renders the geometry inside its view volume to produce a piece of the reflection. Although the reflected objects are modeled with triangle meshes, conventional vertex projection followed by projected triangle rasterization is complicated by several factors. First, a vertex could have more than one projection, even within a single C3RC. Second, the view volume of the $\mathrm{C} 3 \mathrm{RC}$ is complex and triangle clipping is expensive. Third, the edges of the projected triangles are curved, which requires subdividing the triangle such that its projected edges can be acceptably approximated with straight segments. A point-based representation of the reflected objects bypasses these challenges. As a preprocess we sample the reflected geometry and store the points in the kd-tree. At run time the points are splatted using the $\mathrm{C} 3 \mathrm{RC}$ to form the reflection.

The two phase backward-forward approach produces quality reflections at interactive rates, see 
Figure 1, 2, and 5, and the accompanying video. The method has an efficiency advantage over ray tracing because there are two orders of magnitude fewer C3RCs than there are reflected rays, and because the geometry within the view volume of a C3RC is processed efficiently in feed-forward fashion. Compared to a naïve feed-forward approach that renders each triangle with each of the C3RCs, our method benefits from the view volume culling provided by the hierarchical scene subdivision.

\section{PRIOR WORK}

\section{Image-based rendering}

Light fields [LH96, GGS*96] are the most powerful IBR primitive and they naturally support reflections. Light fields can be used to render reflections in one of several ways. The light field can directly provide the desired view ray-it does not matter whether the ray was reflected one or several times on its path from the light source to the eye. Another approach is to use a surface light field [Mil98, WAA*00] attached to the reflector in which to lookup the desired view rays that intersect the reflector. A third possibility is to surround the reflector with a conventionally parameterized light field and to look up the reflected ray, a technique called light field mapping [YM04b]. A fourth approach is based on decoupling reflector properties from illumination. This is achieved with a light field that maps incident rays to reflected rays [Hei99], or to a set of radiance environment maps [CON99].

Several techniques have been developed for rendering reflective surfaces from $2 \mathrm{D}$ ray databases, such as view dependent texture mapping [DYB98], or parameterized environment maps [HAK01]. A disadvantage common to all IBR reflection rendering techniques is the lack of support for dynamic scenes: if the reflecting or reflected objects move, or if the lighting conditions change, the reference rays become obsolete. A second disadvantage is limited support for highly reflective, mirror-like surfaces, which require a high sampling rate and generate impractically large ray databases.

\section{Feed-forward methods}

These methods set out to solve the reflected-point projection problem, in order to make reflection rendering tractable in the context of the feed-forward pipeline. Projection is simple in the case of planar reflectors [Die96], but it does not have a closed form solution in the case of curved reflectors. Explosion maps [OR98] and sample-based cameras [PSM06] tackle the projection problem for curved reflectors. Explosion maps need to be recomputed for every frame, which is inefficient, and the projection of reflected vertices is approximate.

Sample-based cameras are similar to our method, so we describe them in more detail. For each frame, the set of reflected rays is partitioned recursively in sets that are small enough such that they can be approximated well with a planar pinhole camera. An approximation is considered acceptable if the projection error is below a user specified threshold, typically 1 to 5 pixels. The planar pinhole cameras are stored at the leafs of a binary space partitioning tree (BSP), which is called a sample based camera (SBC). The reflection is rendered with the SBC by projecting scene vertices and then by rasterizing the resulting triangles in pure feed-forward fashion. In order to avoid excessive redundancy during the BSP construction and inefficiency during projection, the view frusta of the planar pinhole cameras need to be disjoint. This condition is only satisfied by convex reflectors, which is a severe limitation.

Our method does not require the view frusta of the simple cameras (C3RCs in our case, planar pinhole cameras in the case of SBCs) to be disjoint, since, instead of partitioning the view frusta, we partition the scene to be reflected. The SBC is a compound camera which finds the simple camera that contains a given point in logarithmic time. The first phase of our method on the other hand is similar to ray tracing in that it iterates over all simple cameras and finds the geometry each of them sees in logarithmic time. The advantage is lifting the limiting condition that the reflector be convex.

\section{Ray tracing}

Ray tracing [Whi80, Gla89] is a general rendering technique that produces high quality reflections. In the context of interactive graphics, the challenge is performance. A wide range of acceleration schemes have been proposed, and ray tracing has been shown to run at interactive rates on shared memory parallel computers [Par99], on special hardware [Hal01], on a single CPU [Wal01, WSB01, RSH05], and on GPUs [PBM*02, CHH02, WSE04]. At least for the foreseeable future, GPUs will remain a primarily feed-forward rendering engine. Therefore, techniques like ours that attempt to cast the problem of reflections in terms suitable for feed-forward rendering will continue to best leverage GPUs.

\section{Reflected-scene approximation methods}

First order reflected rays can be easily computed with the feed-forward graphics pipeline: the reflector is processed like any geometry and per-pixel 
reflected rays are computed using per-pixel normals. Per-pixel normals are computed by interpolating vertex normals, by perturbing interpolated normals according to a bump map, or by looking up a normal map. The problem is to intersect the scene with the reflected ray. Several techniques solve this problem by approximating the reflected scene. Environment mapping [BN76, GRE86] is the classic example.

The reflected scene can be modeled with layered depth images (LDIs) [SGH98], and, although ray tracing LDIs is less expensive than ray tracing the entire scene, performance remains an issue [LR98]. A more efficient method models the reflected scene with a sphere of size comparable to size of the environment [Bjo04]. The disadvantage is that the sphere is a crude approximation of the environment, which translates in reflection inaccuracy. A cube map with per-texel depth provides a tighter reflected scene approximation [SALP05]. However, the impostor-ray intersection algorithm makes drastic simplifying assumptions precluding complex scenes.

\section{ALGORITHM OVERVIEW}

Given a scene of reflectors and diffuse nearby objects, the algorithm proceeds as follows.

\subsection{Preprocessing}

In order to support point-based rendering of the reflection, the diffuse geometry is pre-sampled uniformly. One of the challenges of point-based rendering is hole-free reconstruction. One advantage specific to reflections is that the distance from the reflected object to the reflector places a lower bound on the distance between the viewpoint and the pointbased rendered object.

Another advantage is that curved reflectors typically minify the reflected object, which reduces the required sampling rate. Even for concave reflectors there is only a relatively small region of space where rays are condensed. After the quasiconvergence region rays diverge again. When a reflected object is placed in the quasi-convergence region, point-base reconstruction is challenging. Front surface opacity can be maintained by increasing the splat size during rendering. For the examples shown in this paper it was easy to find sampling rates that do not leave holes even for small splat sizes, which ensures a quality reconstruction.

Once the diffuse triangle meshes are sampled, reflections are rendered from the set of diffuse points and the reflector triangle meshes with the following run-time algorithm.

\subsection{Run-time}

\author{
For each frame \\ Build a kd-tree $K$ for the diffuse point set (Section 4). \\ Construct C3RCs for each triangle of each reflector \\ mesh (Section 5). \\ For each C3RC $C$ \\ Intersect view volume $V$ of $C$ into $K$ to find the \\ set $S$ of leafs of $K$ that intersect $V$ (Section 5.2).

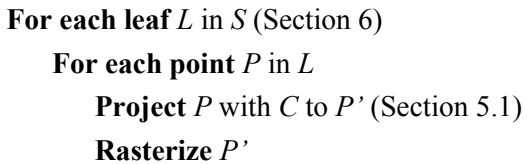

For each leaf $L$ in $S$ (Section 6)

For each point $P$ in $L$

Project $P$ with $C$ to $P^{\prime}$ (Section 5.1)

Rasterize $P^{\prime}$

\section{KD-TREE CONSTRUCTION}

We use a standard kd-tree to organize the diffuse point set hierarchically. The termination criterion for the recursive construction of the tree is reaching a minimum number of points or a minimum size for the leaf node. For simple diffuse objects the kd-tree construction is fast enough to be performed on the fly. Higher complexity diffuse point sets are partitioned off-line. A kd-tree remains valid under rigid body transformations.

\section{CONTINUOUS 3-RAY CAMERA}

C3RCs are the mechanism for taking advantage of reflected ray coherence. A C3RC is built for each reflector triangle, replacing the reflected rays generated by that triangle. A C3RC interpolates between 3 construction rays of unit length [PDM*06]. The 3 rays are the reflected rays at the vertices of the reflector triangle (Figure 3). Given the normal at the vertex and the desired viewpoint, the reflected ray can be easily computed. The C3RC image plane is defined by the tails of the 3 construction rays, thus it coincides with the reflector triangle that generated the $\mathrm{C} 3 \mathrm{RC}$. The $\mathrm{C} 3 \mathrm{RC}$ model is based on the general linear camera model [YM04a], but they have the important advantage of projection continuity across an edge shared by two adjacent C3RCs. In our algorithm C3RCs are called upon to perform two basic operations: 3D point projection and box-view volume intersection.

\subsection{C3RC projection}

The C3RC projection equation is cubic [PDM*06] so a $\mathrm{C} 3 \mathrm{RC}$ can have up to 3 real projections. We 


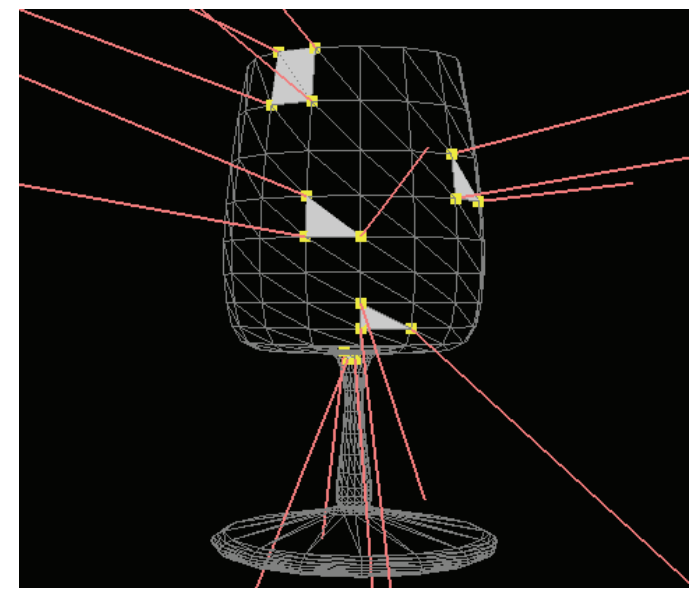

Figure 3: Visualization of sample C3RCs built for a glass shaped reflector. The reflector triangles that define a C3RC are shown in gray. The C3RC construction rays are shown in red.

analyzed C3RC projection in detail to understand if there are cases when more than one of these real projections falls inside the triangular image frame (base) of the C3RC. Such a multiple projection occurs if two or more rays intersect, so a natural approach is to solve the system of equations that searches for a point on two rays. Intersecting two rays yields a fourth order equation. Although closed form solutions exist, it is difficult to decide whether a solution is possible for rays inside the triangular image frame, which has to be tested with inequations on the barycentric coefficients of the rays.

A simpler approach that confirmed the existence of multiple projections is to describe the view volume with a sweeping triangle that cuts equal segments on the construction rays (Figure 4, left). The first sweeping triangle is the C3RC base itself. If a point $Q$ projects inside the base of the $\mathrm{C} 3 \mathrm{RC}$ at
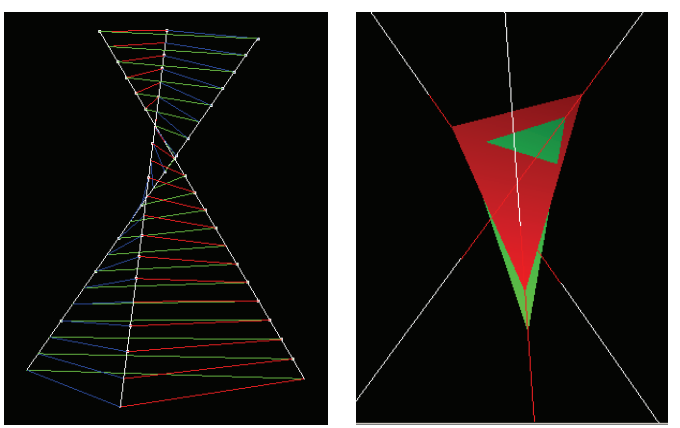

Figure 4: Sweeping triangle visualization of C3RC view volume (left) and self-intersection of sweeping triangle (right). barycentric coordinates $a, b$, and $(1-a-b)$, then there is a position $T$ of the sweeping triangle that contains $Q$ at barycentric coordinates $a, b$, and $(1-a-b)$. If $Q$ is to have two valid projections, there will be two positions of the sweeping triangle that contain $Q$. Figure 4, right shows two intersecting instances of the sweeping triangle. Any point on the intersection segment has at least two valid projections.

The sweeping triangle does not self-intersect for divergent C3RCs, nor does it intersect for all convergent-then-divergent C3RCs like the one seen in Figure 4. We have established a method for deciding whether a C3RC can have multiple projections based on the observation that the sweeping triangle cannot self-intersect while all 3 construction rays are on the same side of the triangle's plane. Initially all construction rays are on the same side of the base. A construction ray switches sidedness when it is contained in the plane of the sweeping triangle.

The sweeping triangle positions where it contains one of the construction rays are found by solving a quadratic equation. If none of three equations has a solution, the construction rays remain on the same side of the sweeping triangle and no multiple projections can occur. If one or more equations have solutions, the view volume subregion where multiple projections can occur is defined by sweeping parameter values in intervals where two construction rays are on one side, and one if on the opposite side of the sweeping triangle. For the example shown in Figure 4, right, multipleprojections can occur when the sweeping triangle intersects the construction rays inside the red segments, which correspond to [0.98, 1.49].

\subsection{Box-view volume intersection}

During the "backward" phase the view volume of each C3RC is intersected with the kd-tree to quickly find the diffuse points inside visible to the C3RC. For this one needs to decide whether a kd-tree node (a box) does or does not intersect the view frustum. A box clearly does not intersect a C3RC frustum if both conditions below are met:

1. The box is not completely inside the frustum.

2. None of the 6 faces of the box intersects any of the 3 side walls of the C3RC frustum.

Testing whether the first condition is satisfied is simply done by testing whether an arbitrary box corner does not have a valid projection.

To test for the second condition, we define the side wall corresponding to base edge $\left(v_{1}, v_{2}\right)$ of a $\mathrm{C} 3 \mathrm{RC}$ with the following parametric surface

$$
\begin{aligned}
S_{1}(\alpha, \lambda) & =(1-\alpha)\left(v_{1}+\lambda d_{1}\right)+\alpha\left(v_{2}+\lambda d_{2}\right) \\
& =v_{1}+\alpha\left(v_{2}-v_{1}\right)+\lambda d_{1}+\lambda \alpha\left(d_{2}-d_{1}\right)
\end{aligned}
$$


where $0<=\alpha<=1$, and $d_{1}$ and $d_{2}$ are the directions of the rays through base vertices $v_{1}$ and $v_{2} . S_{1}$ is intersected with a planar face of the box with corner point $o_{l}$ and normal $n_{l}$ by solving the equation

$$
\left(p-o_{1}\right) n_{1}=0
$$

to find

$$
\lambda=-\frac{v_{1} n_{1}+\alpha\left(v_{2}-v_{1}\right) n_{1}}{d_{1} n_{1}+\alpha\left(d_{2}-d_{1}\right) n_{1}}
$$

Substituting $\lambda$ back into the equation of $S_{l}$, the intersection between the box face and the frustum side wall is determined as a parametric curve $p(\alpha)$

$$
\begin{aligned}
p(\alpha)= & v_{1}+\alpha\left(v_{2}-v_{1}\right) \\
& -\frac{v_{1} n_{1}+\alpha\left(v_{2}-v_{1}\right) n_{1}}{d_{1} n_{1}+\alpha\left(d_{2}-d_{1}\right) n_{1}}\left(d_{1}+\alpha\left(d_{2}-d_{1}\right)\right)
\end{aligned}
$$

The curve $p(\alpha)$ is intersected with the four segments defining the face frame, to determine whether the side wall intersects the box plane inside the face. Each intersection implies solving a quadratic equation.

As always, the box-view frustum intersection is over conservative for the purpose of occlusion culling since the box is assumed to be completely filled with payload geometry. The efficiency of the occlusion culling is analyzed in the results section.

\section{FEED-FORWARD PHASE}

When a kd-tree leaf intersects the view volume of the current $\mathrm{C} 3 \mathrm{RC}$, the points contained by the leaf are projected with a GPU vertex program. Unfortunately the GPU programming framework does not support issuing multiple fragments per vertex, so multiple projections are not supported. The projected vertices are rasterized as square points of fixed size ( 1 or 2 pixels). The reflected scene is three dimensional and proper visibility sorting needs to be enforced. When two points land on the same pixel, the one closer to the reflector surface should win. This is implemented by pushing back a projected point along its desired view ray, similarly to the visibility enforcing mechanism described for sample-based cameras [PSM06]. The push back amount equals the distance from the original diffuse point to the reflector surface, which is given by the distance to the C3RC image plane.

The point-based approach was chosen to avoid the difficulties of reflecting triangle meshes. Consider a diffuse mesh of triangles. Each vertex can have 0 or more projections. The multiple

\begin{tabular}{|c|c|c|c|c|}
\hline Scene & $\begin{array}{c}\text { Points } \\
\mathbf{x 1 , 0 0 0}\end{array}$ & $\begin{array}{c}\text { Constr. } \\
\text { time }[\mathrm{ms}]\end{array}$ & $\begin{array}{c}\text { Avg. } \\
\text { depth }\end{array}$ & $\begin{array}{c}\text { Vertices } \\
\text { per leaf }\end{array}$ \\
\hline Statue0 & 543 & 577 & 7.4 & 122 \\
\hline Dragon0 & 437 & 533 & 7.2 & 121 \\
\hline Bunny0 & 147 & 190 & 6.1 & 121 \\
\hline Dragon1 & 100 & 95 & 5.7 & 122 \\
\hline Bunny1 & 37 & 25 & 4.7 & 121 \\
\hline Dinosaur & 10 & 6 & 3.4 & 123 \\
\hline
\end{tabular}

Table 1. Kd-tree construction performance

projections can originate from the same $\mathrm{C} 3 \mathrm{RC}$, and, more frequently, from different C3RCs. Before rasterization can begin, one needs to examine the projections of the 3 vertices of a given triangle in order to form projected triangles. We have not found a reliable way of grouping vertex projections in projected triangles. Cases when the 3 vertices have a different number of projections or when the projections cannot be easily separated in clusters of triples remain challenging. Moreover, even if the grouping succeeds, conventional rasterization assumes that the edges of the projected triangle are straight, which holds only for small triangles or flat reflectors. The approximation error has to be controlled by subdividing the diffuse geometry, which converges to the point-based approach.

A possible way for bypassing the difficulty of forming reflected triangles is to work within one $\mathrm{C} 3 \mathrm{RC}$ at a time and to clip triangles that extend outside the view volume of the C3RC. Since the side walls of the C3RC are not planar, clipping a triangle is expensive, and the resulting shape is complex, possibly disconnected. Even a triangle completely contained in the view volume of a C3RC can have a complex projection if it crosses the sub-regions where multiple projections occur.

\section{RESULTS}

We have tested the hybrid reflection rendering method on several scenes with good results (Figures 1, 2, and 5, and accompanying video). The background reflection is first rendered by environment mapping and then the reflection of the (synthetic) objects near the reflector is computed with our method.

We have quantified performance for 6 test scenes with variable diffuse geometry complexity. Images of the 6 scenes are shown in row major order in Figure 6. The same 4,000 triangle teapot is used as reflector. All timing data reported in this paper was measured on a $3 \mathrm{~GB} 3.4 \mathrm{GHz}$ Pentium 4 Xeon PC with a Quadro FX 3400 Nvidia graphics card. 


\begin{tabular}{|c|c|c|c|c|}
\hline Scene & $\begin{array}{c}\text { Total } \\
\text { time }[\mathbf{m s}]\end{array}$ & $\begin{array}{c}\text { Culling } \\
{[\mathbf{m s}]}\end{array}$ & $\begin{array}{c}\text { Projection } \\
{[\mathbf{m s}]}\end{array}$ & $\begin{array}{c}\text { Valid proj. } \\
{[\mathbf{\%}]}\end{array}$ \\
\hline Statue0 & 650 & 304 & 346 & 21 \\
\hline Dragon0 & 588 & 273 & 315 & 20 \\
\hline Bunny0 & 264 & 136 & 128 & 19 \\
\hline Dragon1 & 250 & 134 & 116 & 20 \\
\hline Bunny1 & 121 & 63 & 58 & $19 \%$ \\
\hline Dinosaur & 48 & 25 & 23 & $19 \%$ \\
\hline
\end{tabular}

Table 2. Reflection rendering performance.

The performance numbers of the kd-tree construction are given Table 1. For small point sets the kd-tree can be constructed on the fly. Rigid body transformations of the diffuse object do not require re-computing the kd-tree. The powerful eight-way recursion makes the average tree depths and numbers of points per leaf small.

The average hardware projection performance is 3.4 million vertices per second. The performance of each of the two phases of reflection rendering is given in Table 2 for each of the 6 test scenes. A
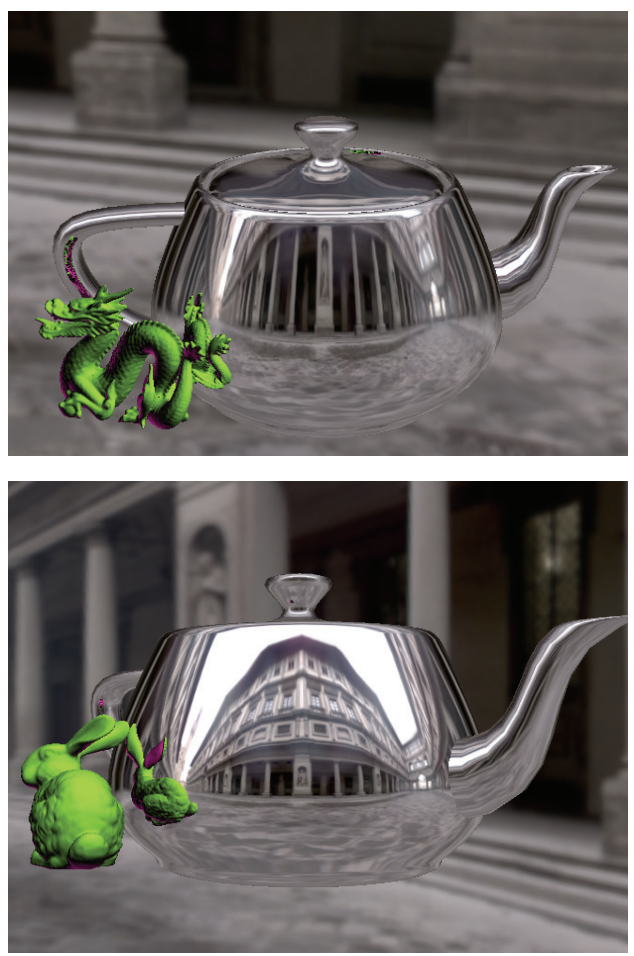

Figure 5: Reflections of dragon and bunny rendered with our method over an environment mapped background. frame rate of $4 \mathrm{fps}$ or better is obtained on all but the two most complex diffuse scenes. Appropriate parameters need to be chosen in the kd-tree construction to balance the computation burden between software culling and hardware projection. A deep kd-tree and small leaf nodes accelerate projection by increasing the ratio of valid projections at the expense of slowing down view volume culling. On the other hand a shallow tree and large leafs reduce the culling effort at the expense of a large number of unnecessary projections. The table shows that for our system a good balance between the two phases was obtained for a ratio of valid projections of about $20 \%$.

The performance of the method depends on the complexity of the reflector in two ways. The number of C3RCs equals the number of reflector triangles, so the number of times the two rendering phases are executed is linear in the number of reflector triangles. The reflector shape complexity also greatly influences performance. High curvatures generate $\mathrm{C} 3 \mathrm{RCs}$ with wide open view volumes, which project a large number of points. For the teapot for example, the handle, spout, and lid knob project almost every point. Consequently most points have on average 4 projections. Since the knob is small in screen space, it should be rendered with environment mapping.

\section{CONCLUSIONS}

Hybrid backward-forward reflection rendering produces good results at interactive frame rates. The versatility of points was leveraged to overcome difficulties that arise in rendering reflected meshes. This paper also furthers the understanding of continuous 3-ray cameras which are important infrastructure for applications beyond reflections.

Compared to ray-tracing, our technique is faster if forward rendering of the geometry inside the $\mathrm{C} 3 \mathrm{RC}$ view volume is faster than ray tracing the same geometry. This is the case if the reflector triangle covers sufficient pixels in screen space. If the reflector triangle covers only a few pixels, feedforward rendering does not pay off since the setup cost is not amortized over enough pixels. Compared to IBR techniques, our method has the advantage of supporting highly specular surfaces and moving objects, whereas IBR techniques can render scenes with unknown geometry. Compared to reflectedscene approximation techniques, our method is more accurate, at the expense of performance.

Probably the best avenue for increasing performance is to provide hardware support for the C3RC. The projection is constant time, but the 

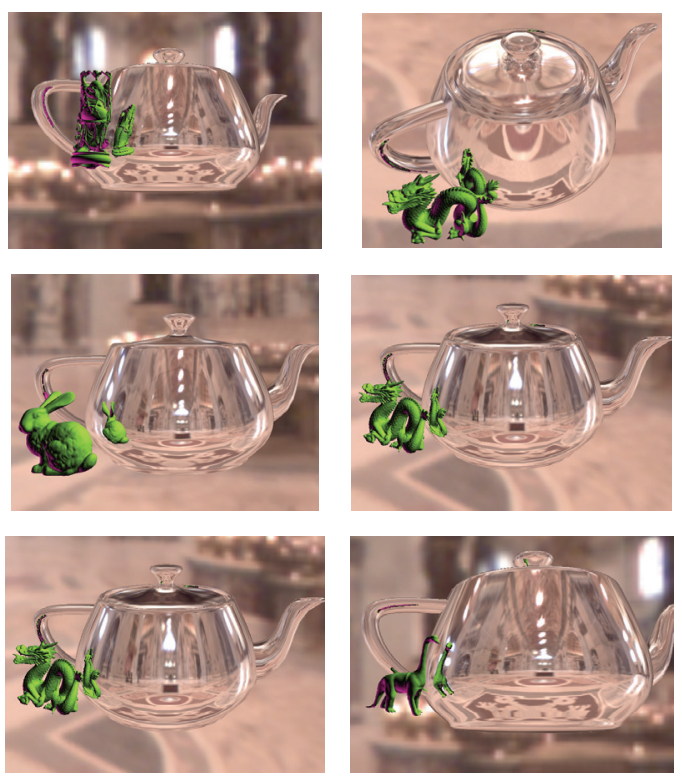

Figure 6: Scenes used to measure performance.

constant is rather large, requiring solving a cubic. Dedicated hardware could make C3RC projection as efficient as conventional perspective projection. Software view-frustum culling is equally expensive, and we will investigate approaches for traversing the $\mathrm{kd}$-tree in the vertex program.

\section{ACKNOWLEDGMENTS}

This work was supported by the United States National Science Foundation through grants SCI0417458 and CCR-9617600.

\section{REFERENCES}

[Ben79] BENTLEY, J. L.: Data structures for range searching. Computing Surveys, 11(4), December 1979.

[Bjo04] BJORKE K.: Image-based lighting. GPU Gems, Fernando R., (Ed.). NVidia, (2004), pp. 307-322.

[BN76] BLINN J.F., NEWELL M.E.: Texture and Reflection in Computer Generated Images. CACM 19:10, 542-547, 1976.

[CON99] CABRAL B.: OLANO M., NEMEC P.: Reflection Space Image Based Rendering. In Proc. of SIGG '99, pp.165-170. [CHH02] CARR N., HALL J.D., HART J.C. The Ray Engine, Graphics Hardware (2002), pp. 1-10

[DYB98] DEBEVEC P., YU Y., BORSHUKOV G.: 1998 Efficient view-dependent image-based rendering with projective texture-mapping. In Proc EGWR, 105-116.
[Die96] DIEFENBACH P. J.: Pipeline Rendering: Interaction and Realism Through Hardware-Based Multi-Pass Rendering. $\mathrm{PhD}$ thesis, University of Pennsylvania, (June 1996).

[Gla89] GLASSNER, A. An introduction to ray tracing. Academic Press, 1989.

[GRE86] GREENE, N. Environment mapping and other applications of world projections. IEEE CG\&A, 6:11, (1986).

[Hal01] HALL D.: The AR350.

[HAK01] HAKURA Z.: Parameterized Environment Maps. In Proc. of ACM I3D 2001 (2001), pp 203-208.

[HM92] HANRAHAN P., MITCHELL D.: Illumination from curved reflectors. In Proc. of SIGG '92, ACM Press, pp. 283-291.

[Hei99] HEIDRICH W.: Light Field Techniques for Reflections and Refractions. EGRW 1999 (1999), pp.195-375.

[LH96] LEVOY M., HANRAHAN P.: Light Field Rendering. Proc. of SIGGRAPH 96 (1996), 31-42.

[LR98] LISCHINSKI D., RAPPOPORT A.: Image-Based Rendering for Non-Diffuse Synthetic Scenes. Eurographics Rendering Workshop 1998 (1998), pp.301-314.

[Mil98] MILLER G.: Lazy Decompression of Surface Light Fields for Precomputed Global Illumination, Eurographics Workshop on Rendering 1998 (1998).

[OR98] OFEK E., RAPPOPORT A.: Interactive reflections on curved objects. In Proc. of SIGGRAPH '98, ACM Press, 333-342.

[Par99] PARKER, S.: Interactive ray tracing. ACM Symposium on Interactive 3D Graphics (1999), 119-126.

[PDM*06] POPESCU, V., DAUBLE J., MEI, C., and SACKS, E.: An Efficient Error-Bounded General Camera Model. In Proc. of Third International Symposium on 3D Data Processing, Visualization, and Transmission (2006).

[PSM06] POPESCU V., SACKS E., and MEI C.: Sample-Based cameras for feed forward reflection rendering. IEEE Transactions on Visualization and Computer Graphics, (2006), to appear.

[PBM*02] PURCELL T.J., BUCK I., MARK W. Ray Tracing on Programmable Graphics Hardware, In Proc. of SIGG '02 (2002).

[RSH05] RESHETOV A., SOUPIKOV R., HURLEY J.: MultiLevel Ray Tracing Algorithm, In Proc. of SIGG 2005 (2005).

[SGH98] SHADE J., GORTLER S., HE L., SZELISKI R.: Layered Depth Images, In Proc. of SIGG 98 (1998), 231-242.

[SALP05] SZIRMAY-KALOS L., ASZODI B., LAZANYI I., PREMECZ M.: Approximate Ray-Tracing on the GPU with Distance Impostors. EUROGRAPHICS 2005 24, 3.

[WSB01] WALD I., SLUSSALEK P., BENTHIN C.: Interactive distributed ray tracing of highly complex models. In Rendering Techniques 2001: 12th EGWR (2001), 277-288.

[Wa101] WALD I.: Interactive rendering with coherent ray tracing. Computer Graphics Forum 20, 3 (2001), 153-164.

[WSE04] WEISKOPF D., SCHAFHIZEL T., ERTL T. GPUBased Nonlinear Ray Tracing, In Proc. of EG '04 (2004).

[Whi80] WHITTED T.: An improved illumination model for shaded display. Comm. Of the ACM (1980), 23, 6, pp. 343-349.

[WAA*00] WOOD D.N., AZUMA D. I., ALDINGER K.: Surface light fields for 3D photography. In Proc. of SIGG '00, pp. 287-296.

[YM04a] YU, J., and McMILLAN, L.: General Linear Cameras In 8th European Conference on Computer Vision (ECCV), 2004, Volume 2, 14-27.

[YM04b] YU, J. and McMILLAN, L.: Real-time reflection mapping with parallax. In Proceedings of ACM Symposium on Interactive 3D Graphics and Games (I3D), 2005. 